\title{
Special Section Introduction: Elastography
}

\author{
Alvin C. Silva, ${ }^{1}$ Michael D. Beland ${ }^{2}$ \\ ${ }^{1}$ Department of Radiology, Mayo Clinic, Scottsdale, AZ 85255, USA \\ ${ }^{2}$ Department of Diagnostic Imaging, Rhode Island Hospital, Providence, RI 02903, USA
}

Elasticity is a physical property of an object or material to revert to its normal shape after being deformed. Elastography is a novel imaging method to detect and quantify pathologic processes that are known to alter the elastic properties of normal tissues in the body, providing previously unavailable non-invasive insight to disease that can directly impact patient assessment and management.

Currently, elastography is performed with either ultrasound (US) or magnetic resonance (MR) imaging. In this special focus edition, Garra provides an overview of the history and development of US elastography, also referred to as sonoelastography, and compares and contrasts the two currently applied methods: strain and shear wave elastography. Dahyani and colleagues further review specific applications for shear wave elastography in the liver-including evaluating focal masses, diffuse diseases, and assessing portal hypertension. Zhang et al. prospectively evaluated the performance of shear wave elastography versus aspartate-to-platelet ratio index (a commonly accepted non-invasive liver fibrosis test) using liver biopsy as the reference standard, whereas Cabassa et al. investigated the correlation between liver fibrosis and shear wave spleen stiffness measurements in chronic viral hepatitis patients. Finally, Altiparmak et al. evaluated the diagnostic value of two strain ratios (intrahepatic vein-to-liver parenchyma and intercostal muscle-to-liver parenchyma) for determining liver fibrosis.

For MR Elastography (MRE), Venkatesh and colleague provide an overview of the technical and analytical approaches for this technique, as well as discuss clinical applications in the liver, other solid abdominal organs, and the uterus. Batheja et al. investigated the performance of MRE for hepatic fibrosis in a cohort of diseased and normal livers, all of whom had histologic correlation, whereas Gallegos-Orozco et al. evaluated the efficacy of MRE for discriminating normal from abnormal biopsy results in liver donor candidates. In addition, the manuscripts in this focus edition also attempt to answer specific practical questions related to MRE:

1. How does MRE compare to accepted morphologic imaging criteria for liver fibrosis and cirrhosis? (Venkatesh et al.)

2. Are there stiffness variations for the different hepatic segments in a normal liver? (Rusak et al.)

3. What is the effect of gadolinium contrast on liver stiffness? (Hallinan et al.)

4. Which ROI measurement method is better, single versus triple? (Silva et al.)

5. Are the results reproducible across different vendors? (Serai et al.)

As MRE is still in its relative infancy, there continue to be evolving technical advancements. Dyvorne et al. explored the feasibility of using dual acoustic drivers for simultaneous liver and spleen assessment for evaluating liver disease severity and portal hypertension. Silva et al. investigated the effect of an improved inversion algorithm on currently established hepatic stiffness values for staging liver fibrosis, and showed that the use of the new confidence threshold mask reduces intraobserver and interobserver calculated stiffness variability. Finally, Guo et al. presented the results of a meta-analysis comparing the performance of MRE (11 studies; 982 patients) versus shear wave elastography (15 studies; 2128 patients).

We hope you will enjoy this special section and that you will incorporate elastography appropriately into your practice.

Correspondence to: Alvin C. Silva; email: Silva.Alvin@mayo.edu 